\title{
Author Index to Volume 87
}

\section{$\mathbf{A}$}

Aarsland, Sara J. 128

Abai, Mohammad R. 499

Abalos, Rodolfo M. 327

Abamor, Emrah Sefik 81

Abbasi, Ibrahim 883, 1059

Abbey, Mercy S11

Abdelgader, Tarig M. 1012

Abiola, Annie 640

AbouLaila, Mahmoud 681

Abuaku, Benjamin K. 996

Accrombessi, Manfred M. K. 418

Acuna-Retamar, Mariana 473

Adam, Ishag 35

Adiossan, Lukas G. 425

Adjuik, Martin S11

Afwamba, Isaac A. 1105

Agudelo, Carlos A. 1094

Aguirre, Eglys 538

Ahmad, Norazah 737

Ahmed, Salwa F. 312

Ahuja, Anil A. 261

Aikins, Moses S11

Ainsworth, Robert M. 11

Ajdary, Soheila 70

Akoko, Daniel 385

Alaii, Jane 1065

Alain, Michault 134

Albertini, Audrey 437

Aldana, Rodrigo 837

Alexandre, Márcia A. A. 1119

Alfven, Tobias S36

Alifrangis, Michael 640

Aliganyira, Patrick S46

Alilio, Martin 960

Alimohammadian, Mohammad H. 70

Allahverdiyev, Adil M. 81

Allan, Richard 242, 407

Almeida, Igor C. 87

Almeida, Marcos de 297

Alonso, Diego P. 470

Alonso, Pedro 1119

Alphonsus, Kal M. 272

Alva, Isaac E. 954

Alvar, Jorge 1028

Alvarez, Mayling 538

Alvarez-Manzaneda, Enrique 481

Amendola, Paul S144

Amenga-Etego, Lucas S21

Amico, Peter 581

Amine, Ahmed A. A. 1012

Anders, Katherine L. 165

Anderson, John F. 754

Andersson, Sarah S120

Andrade, Zilton A. 843

Andreasen, Aura 868

Añez, Germán 548

Anthony Lake S1

Anyorigiya, Thomas S21

Aoki, Valeria 675

Appanna, Ramapraba 936

Arevalo-Herrera, Myriam 460

Argaw, Daniel 1028

Argüello, Ana Patricia 345

Arguin, Paul 192

Armstrong, Philip M. 754

Arora, Ankur 1

Arrospide, Nancy 491

Assis, Rafael R. 87

Avila-Figueroa, Carlos 581
Awini, Elizabeth S11

Awiti, Alphonce 1065

Awor, Phyllis S92

Awuondom, Ken 1004

Ayode, Desta 623

Azeredo-Coutinho, Rilza Beatriz 257

\section{B}

Bacigalupo, Antonella 473

Bagirova, Malahat 81

Bai, Ying 518

Baird, J. Kevin 41

Bakhiet, Sahar 1012

Balagon, Maria V. 327

Baldeviano, G. Christian 511

Banda, Rose 57

Bang, Heejung 868

Bannicq, Marika Yip S85

Baptista, Cibele 257

Barakat, Maha 701

Barat, Lawrence M. S69

Barbosa, Lúcio M. 843

Bari, Abdul S137

Barnish, Guy S21

Bartlett, John A. 1105

Bartsch, Sarah M. 616

Bassat, Quique 1119

Basseri, Hamid R. 499

Basso, César 1083

Bastos, Melissa da Silva 813

Bastos, Michele S. 1119

Basu, Roshni S85

Bates, Arturo 1132

Bayona, Jaime 399

Beare, Nicholas A. V. 985

Bebes, Sauli 1145

Beeche, Arlyne 242

Begum, Yasmin Ara 921

Bell, David 57, 437

Benítez-Ortíz, Washington 524

Bennett, Adam 437

Benton, Lorna S11

Bern, Caryn 1028

Bernabe-Ortiz, Antonio 954

Betson, Martha 694

Bhattacharya, Sujit K. 1028

Bieri, Franziska A. 972

Biggs, Holly M. 1105

Bilbao, Juan 715

Bingham, Andrea M. 1140

Binkin, Nancy S85

Bishop, Henry 297

Bishop, Henry S. 1073

Blacksell, Stuart D. 171, 281, 573

Blackwell, Jenefer M. 663

Blank, Walter A. 843

Blanton, Ronald E. 843

Blas, Magaly M. 954

Blitvich, Bradley J. 1132

Bodeau-Livinec, Florence 418

Boehme, Catharina C. 202

Boel, Machteld E. 447

Bojórquez-Contreras, Ángel Noel 888

Bonamigo, Raquel A. 470

Boniface, Karen 1145

Bonilla, Cesear 399

Boqvist, Sofia 1076

Bosco-Lauth, Angela 760
Botto-Mahan, Carezza 473

Boubour, Jean 602

Bougnoux, Marie-Elisabeth 303

Bouley, Andrew J. 1105

Bourhy, Pascale 134

Bowen, Richard 760

Bowman, Natalie M. 1022

Braga, Wornei Silva Miranda 768

Brandariz, Silvia B. 267

Brandt, Jef 524

Brault, Aaron C. 559

Breman, Joel G. 231

Bricaire, François 1041

Briggs, Caitlin M. 743

Brisson, Michael 959

Brisson, Paul 959

Brooker, Simon J. 1004

Brown, Dennis T. 743

Brown, Joseph 394

Bruton, Jody A. 312

Bryce, Jennifer S61, S127

Buckendahl, John 125

Buffardi, Anne L. 954

Buffet, Pierre 435

Bumb, Ram A. 261

Burchard, Gerd D. 190

Burke, Nicholas 960

Burkett-Cadena, Nathan D. 1140

Burkhalter, Kristen 1125

Burns, Matthew 242

Bustos, Javier A. 292

Butler, Sara E. 862

Bwire, Godfrey S92

Bytzer, Peter 1046

\section{C}

Cabada, Miguel M. 128

Cabezas, Cesar 491

Caffera, Ruben M. 1083

Cakir-Koc, Rabia 81

Caldas, Adolfo 399

Calderwood, Stephen B. 921

Calheiros, Cláudia M. L. 125

Calisher, Charles H. 1132

Callaghan-Koru, Jennifer A. S54, S61, S127

Campista-León, Samuel 888

Campos, Xavier 381

Canim-Ates, Sezen 81

Cao, Xinghua 104

Carcamo, Cesar P. 954

Cardemil, Cristina V. S127

Cárdenas, Ángela 837

Cárdenas, María Kathia 197

Cardinale, Eric 134

Carlton, Elizabeth J. 1053

Carneiro, Ilona 242

Carnevale, Pierre 242

Carrasco, Luis R. 1116

Carrillo, Lina 565

Carroll, Brian D. 559

Carstensen, Suzana 371

Carter, Yvonne L. 1038

Carvalho, Ricardo C. 1119

Castellanos-Gonzalez, Alejandro 128

Cataño, Juan Carlos 195

Cattan, Pedro E. 473

Caumes, Eric 435, 1041

Cellona, Roland V. 327 
Chahboun, Rachid 481

Chandani, Yasmin S120

Chang, Aileen Y. 616

Chang, Chia-Ming 141

Chang, Ko 141

Chang, Litao 185

Chang, Shu-Fen 349

Changalucha, John M. 868

Chansamouth, Vilada 171

Chanthongthip, Anisone 573

Chao, Chien-Chung 148

Chao, Chien-Ming 933

Charles, Richelle C. 921

Charoenmak, Boonsri 927

Chau, Nguyen Van Vinh 165

Cheek, James E. 529

Chen, Mark I-C 1116

Chen, Tun-Chieh 141

Chen, Yen-Hsu 141

Chen, Yingdan 97

Cheng, Xunjia 97

Chiappe, Marina 954

Chimuna, Tiyese S77

Chinbuah, Margaret A. S11

Ching, Wei-Mei 148

Chirico, Jan 1076

Chisha, Zunda 437

Chowdhury, Fahima 921

Christiansen, Gina 153

Chunn, Kuan Yeh 737

Chusri, Sarunyou 76, 927

Ciuoderis, Karl A. 565

Clair, Bernard 435

Clarysse, Guy S85

Cleaveland, Sarah 1105

Clements, Archie 1153

Cline, Joanne 312

Conlan, James V. 281

Conn, Jan E. 910

Consolaro, Marcia E. L. 1149

Conteh, Lesong S111

Cook, Alex R. 1116

Copenhaver, David J. 616

Cordray, Michael S. 223

Cornelie, Sylvie 504

Correa, Margarita M. 910

Corstjens, Paul L. A. M. 868

Cortes, Jose A. 781

Corwin, Andrew 965

Costa de Oliveira, Cintia Mara 768

Cot, Michel 418

Cotrina, Armando 954

Counihan, Helen 57, S46

Cournil, Amandine 504

Cousens, Simon S21

Cox, Nancy 965

Cramer, Jakob P. 190

Cristaldi, Flávia 675

Crump, John A. 1105

Cruz, Israel 1028

Cruz, Maria do Socorro Pires e 470

Cruz-Zetina, Guillermo 671

Cucunubá, Zulma M. 837

Cui, Feng 185

\section{D}

D’Alessandro, Umberto 251, 989

D'Andrea, Paulo S. 371

D'Harcourt, Emmanuel S144

da Costa Campos, Lenilton 741

da Costa Castilho, Márcia 768

da Costa, Paulo Sérgio Gonçalves 741

da Rosa, Amelia Travassos 1132 da Rosa, Elsa García 1083

da Silva, Alexandre J. 297

da Silva, Mariana C. 1149

Daelmans, Bernadette S54

Dahlgren, F. Scott 529

Dama, Souleymane 23

Dara, Antoine 23

Darabi, Haideh 70

Dat, Nguyen Tan 554

Datta, Siddhartha Sankar 1145

Daulaire, Nils 382

Davaasuren, Batdorj 681

Davey, Gail 623

Dayan, Gustavo H. 584

de Abreu, André L. P. 1149

de Araujo, Marina R. 371

de Borba, Luana 371

de Camargo, Zoilo Pires 710

de Dood, Claudia J. 868

de Freitas, Elder Lanzani 675

de Groot, Philip G. 943

de Heer, Hendrik 623

de Hoog, G. Sybren 710

De Los Santos, Maxy B. 511

de Mast, Quirijn 943

de Oliveira, Alessandra Gutierrez 470

de Oliveira, Raquel de Vasconcellos Carvalhaes 257

De Silva, Nalika 342

de Sousa, Alexandra S85

Del Brutto, Oscar H. 381

del Diego, Jorge 584

del Rio-Rodriguez, Rodolfo 671

Dela Cruz, Eduardo C. 327

Delfraro, Adriana 371

Delgado, Livia 675

Delgado-Vargas, Francisco 888

Delorey, Mark 1125

Delorey, Mark J. 1112

Dembele, Demba 23, 50

den Boer, Margriet 1028

Denis, Blandine 303

Dent, Arlene E. 806

Desai, Mayur M. 979

Desvars, Amélie 134

Diakite, Bamody S77

Diara, Malick 960

Diaz, Luis A. 675

Diaz, Theresa S85

Díaz-Camacho, Sylvia Páz 888

Dick, Olivia Brathwaite 584

Dicko, Alassane 50

Dickson, Sammie L. 359

Dieng, Yemou 640

Ding, Lei 1099

Ding, Shu-Jun 1099

Djamiatun, Kis 943

Djimde, Abdoulaye A. 23, 50

Dondorp, Arjen M. 231

Dorny, Pierre 281

dos Santos, Claudiney Biral 1089

Douce, Richard 524

Doucoure, Souleymane 504

Doumbo, Ogobara K. 23, 50

Dournon, Nathalie 435

Downs, Jennifer A. 868

Duah, Nancy O. 996

Duarte dos Santos, Claudia N. 371

Duc, Hoang Minh 554

Dunavan, Claire Panosian 193, 775

Duncombe, Jennifer 1153

Dung, Nguyen Thi 165

Duong, Socheat 231

Duplisea, Kevin 715
$\mathbf{E}$

Eaton, Donald P. 675

Eberhard, Mark L. 1073

Edgel, Kimberly A. 511

Edupuganti, Srilatha 1112

Eigege, Abel 272

Eisele, Thomas P. 437

Eisen, Lars 902

Ekpo, Pattama 148

Ekram, A. R. M. Saifuddin 658

Elcicek, Serhat 81

Elimelech, Menachem 979

Elliot, Aileen 281

ElMardi, Khalid A. 1012

El-Mohammady, Hanan 312

Elyazar, Iqbal R. F. 41

Engsbro, Anne Line 1046

Epelboin, Loïc 1041

Erhart, Annette 989

Ernst, Kacey C. 806

Esch, Kevin J. 822

Espiau, Maria 832

Espina, Luz M. 548

Espino, Fe Esperanza 1153

Espiritu, Betty 399

Evangelista, Wilfredo 153

\section{F}

Fairhurst, Rick M. 231

Faith, Sitnah Hamidah 594

Fajardo, Tranquilino T. 327

Falqueto, Aloísio 1089

Fan, Desheng 185

Fan, Victoria Y. 579

Faradz, Sultana M. H. 943

Farajollahi, Ary 359

Farfán-Ale, José A. 1132

Faye, Babacar 640

Fedak, Kristen 117

Feldmeier, Hermann 125

Felgner, Philip L. 460

Feng, Youmei 689

Fenwick, Stanley 281

Fernandez, Roberto 511

Fernando, Champika 495

Ferreira, Adelson Luiz 1089

Ferreira, Alda M. T. 470

Ferreira, Érika C. 1149

Ferreira, Gabriel Eduardo Melim 1089

Ferreira, Marcelo U. 813

Ferrer, Patricia 153

Fidock, David A. 16

Figueiredo, Regina M. P. 1119

Figueras, Concepción 832

Fijnheer, Rob 943

Fink, Günther 608

Finney, Olivia C. 460

Fischer, Marc 179, 1112

Fischer, Peter U. 491

Fitzgerald, Daniel W. 868

Fleming, Michael 616

Flores, Jorge Vargas 504

Flores, Myra 153

Flórez, Astrid C. 837

Fofana, Bakary 23, 50

Folkema, Arianne M. 529

Fonseca, Dina M. 719

Foster, Woodbridge A. 727

Freeman, Matthew C. 385

Freitas, Gustavo F. 87

Fuad, Anis 1152 
Fuentes, Patricia 153

Fung, Mai S. 1053

\section{G}

Gamache, Nathalie S46

Gamboa, Ricardo 897

Gan, Tran Thi 165

García, Alejandro 473

García, Gissel 538

Garcia, Hector H. 292, 897

García, Patricia J. 954

Garcia-Rejon, Julian E. 1132

Gardner, Malcolm J. 460

Garg, Hitendra 1

Gau, Shiow-Jen 933

Gaudart, Jean 50

Gautam, Shalini 655

Gaye, Oumar 640

Gebreyesus, Tsega 623

George, Asha S61, S69, S85

Gibbons, Robert V. 171

Gibney, Katherine B. 1112

Gilbert, Amy T. 206

Gilbert, Amy Turmelle 518

Gilbos, Valery 407

Gilman, Robert H. 153, 197, 292

Gilroy, Kate S54, S69

Gilroy, Kate E. S61, S127

Gimaque, João B. L. 1119

Gimaque, João Bosco de Lima 768

Gimenes, Fabrícia 1149

Giorgi, Roch 50

Giraldez, Mabel Guerra 504

Gitonga, Caroline W. 1004

Glassman, Amanda 579

Glinz, Dominik 425

Glover, Simon J. 985

Godsey, Marvin S. Jr. 1125

Gómez, Jorge 206, 518

Gómez, Karina A. 267

Gonzalez, Armando E. 292

González, Cristina 1083

Graf, Paul C. F. 511

Graham, Sean P. 1140

Graves, Patricia 272

Graves, Sonya E. 359

Gray, Darren J. 972

Greene, Leslie E. 385

Griffioen, Arjan W. 943

$\mathrm{Gu}$, Meilin 104

Guenther, Tanya S46, S69, S77

Guimarães, Isabel C. 843

Guiot, Marie-Christine 715

Gundi, Vijay A. K. B. 319

Guo, Na-Na 364

Gutiérrez, Andrés H. 153

Gutiérrez, Lina A. 910

Gutierrez-Sánchez, Ramón 481

Guzmán, María G. 538

Gyapong, John O. S11

Gyapong, Margaret S11

\section{H}

Halasa, Yara 796

Hall, Rebecca L. 297

Hallett, Rachel 231, 640

Hamainza, Busiku 57, 437, S105

Hamburger, Joseph 883, 1059

Hamer, Davidson H. S2, S69, S105, S151

Hammamy, Diaa S6, S69

Hammond, Chris 297
Hamoudi, Amar 18

Han, Xu 364

Han, Zhan-Ying 364

Hans-Filho, Günter 675

Harding, Simon P. 985

Harmen, Sonia 960

Harris, Jason B. 921

Harris, Julie R. 594

Harshfield, Eric 786

Harvey, Steven A. 57

Hashiguchi, Yoshihisa 658

Hassan, Hassan K. 1140

Hawk, Douglas 312

Hayden, Mary H. 902

Heisey, Daniel A. R. 548

Hernandez, Raquel 743

Herrera, Socrates 460

Herve, Jean Pierre 504

Heukelbach, Jörg 125

Hill, Allan G. 608

Hill, David R. 11

Hill, Janet E. 495

Hill, Zelee S111

Hin, How Soon 737

Hoekstra, Robert M. 594

Hoffman, Richard S. 1073

Hofmeister, Erik 565

Holman, Robert C. 529

Horie, Toshiharu 64

Hortiwakul, Thanaporn 76, 927

Horwood, Paul Francis 1145

Hosseini, Zohreh 70

Hotez, Peter J. 3

Hsieh, Hsiao-Chen 141

Hsu, Chien-Yeh 1152

Hsu, Tung-Chien 349

Hu, Wenbiao 1153

Huang, Jyh-Hsiung 349

Hübscher, Tanja 573

Humberg, Roberta M. P. 470

Hun, Laya 345

Hung, Le Xuan 989

Hurrell, Richard F. 425

Hutchinson, Michael 359

Huu, Le Minh 554

Huynh, Trang T. T. 631

Hyde, Molly 874

Hyder, Adnan A. S61

Ibad-ul-Haque S137

Ibrahim, Naglaa 701

Ichikawa, Madoka 681

Ichimura, Ligia Maria F. 675

Igarashi, Ikuo 681

Imtawil, Kanokwan 706

Incardona, Sandra 437

Intapan, Pewpan M. 706

Ireri, Edmund 874

Islam, Md. Anwar Ul 658

Islam, Mohammad Zahidul 658

Itoh, Makoto 658

Iyer, Hari S105

\section{$\mathbf{J}$}

Jackson, Bryan T. 727

Jagoe, George S92

Jaramillo, Luz M. 910

Jarman, Richard G. 171

Jauréguiberry, Stéphane 435

Jave, Oswaldo 399
Jenkins, Emily J. 495

Jeronimo, Selma M. B. 663, 822

Jeuland, Marc 18

Jimenez, Juan A. 292, 897

Jin, Faguang 947

Jin, Yuming 185

Jip, Nimzing 272

Jiya, Jonathan 272

John, Chandy C. 806

Johnson Jr., Warren D. 868

Jones, Jeffrey L. 297

Jones, Stephen L. 70

José Rosales, María 481

Juliano, Jonathan J. 1038

Juliao, Patricia 594

Junior, Cleber Soares 741

\section{$\mathbf{K}$}

Kabatereine, Narcis B. 694

Kachule, Timothy S54

Kadobera, Daniel S30, S36

Kager, Piet A. S11

Kalilani-Phiri, Linda 29, 1022

Källander, Karin S21, S46, S97, S111

Kalluvya, Samuel E. 868

Kalyango, Joan N. S30, S36

Kamara, Sarian 242

Karamagi, Charles S36

Karanja, Diana M. S. 862, 874, 1065

Kariuki, Curtis 1059

Kasai, Takeshi 965

Kaseman, Tremayne 602

Kattenberg, Johanna H. 251

Katureebe, Sheila S30

Katz, Alan R. 337

Kauffman, Carol A. 1094

Kay, Brian H. 554, 631

Kayemba, Christine Nalwadda S46

Keating, Joseph 437

Keereecharoen, Naw Lily 447

Kerber, Kate S46

Kesavaraju, Banugopan 359

Kesper Jr., Norival 675

Ketmayoon, Pakapak 965

Khamlome, Boualam 281

Khamphaphongphanh, Bouaphanh 965

Khan, Amanullah S137

Khan, Ashraful I. 921

Khan, Attaullah S137

Khanthamaly, Viengphone 965

Kihara, Jimmy H. 1004

Kilian, Albert 407

Kim, Dohyeong 117

Kimura, Eisaku 658

Kinabo, Grace D. 1105

King, Charles H. 1059

King, Jonathan D. 272

Kirkwood, Betty S111

Kirkwood, Carl Dunn 1145

Kirwan, Daniela E. 197

Ko, Wen-Chien 141

Kobylinski, Kevin C. 902

Kochel, Tadeusz J. 954

Kohler, Dennis 760

Kola, Steve 594

Kolyvas, George 715

Konaté, Amadou T. S21

Kone, Aminatou 23

Koram, Kwadwo A. 996

Korevaar, Daniel Arnoldus 583

Kosoy, Michael Y. 319, 518

Kosoy, Olga I. 1112

Koua, Ahou-Yah G. 425 
Kounnavong, Bounheuang 965

Koura, Ghislain K. 418

Kramer, Randall 117

Krasavin, Nina M. 359

Kronmann, Karl C. 996

Kulkarni, Rajan P. 616

Kutcher, Simon 554, 631

\section{$\mathbf{L}$}

Lacerda, Marcus V. G. 1119

Lafon, Sonia O. 267

Laguna-Torres, V. Alberto 206

Lai, Chih-Cheng 933

Lai, Chung-Hsu 141

Lai, Ping-Chang 141

Laínez, Yolanda Barberá S144

Lairihoy, Rosario 1083

Lamba, Doriano 153

Lampman, Richard L. 359

Lanciotti, Robert S. 1112

Landa-Piedra, Abraham 888

Langevin, Stanley A. 559

Lantagne, Daniele 786

Lanternier, Fanny 303

Largusa, Ron 297

LaRocque, Regina C. 921

Laudisoit, Anne 319

Lazuardi, Lutfan 1152

Le Goff, Gilbert 504

Leão, Jorge Di Tommaso 768

Lecuit, Marc 303

Lee, Bruce Y. 616

Lee, Hsin-Chun 141

Lee, Nan-Yao 141

Lee, Sue J. 171, 573

Lee, Vernon J. 1116

Lehman, Jennifer A. 179

Leite, Gustavo Rocha 1089

Lemos, Elba R. S. 371

Leo, Yee Sin 1116

Lescano, Andrés G. 511

Leshem, Eyal 264

Leung, Daniel T. 921

LeVasseur, James 565

Levin, Mariano J. 267

Levy, Craig 1125

Lewallen, Susan 985

Lewis, Hannah 965

Li, Dongmei 337

Li, Guohua 185

Li, Haitao 646

Li, Jiayin 306

Li, Liang 185

Li, Qi 364

Li, Weihong 185

Li, Xiaobo 947

Li, Yimei 104

Li, Yue-Sheng 972

Li, Zhong 1099

Liang, Xiaowu 460

Liao, Tsai-Ling 349

Lin, Chien-Chou 349

Lin, Chun-Yu 141

Lin, Wei-Ru 141

Lindahl, Johanna 1076

Lindblade, Kim A. 806

Lindegardh, Niklas 35

Lindsay, Ann 297

Lindsey, Nicole P. 179

Lindstrand, Ann S36

Lindtjørn, Bernt 450

Liu, Hong 185

Liu, Tao 104

Liu, Ying-Ying 364
Liu, Zaoling 104

Liu, Zhaoyan 306

Liyanapathirana, Veranja 342

Lo, Aminata 640

Loayza, Roxanna 504

Lockamy, Kameron P. 128

Loha, Eskindir 450

Lombardo, Sarah 18

Long, Yong 646

Longhi, Silvia A. 267

Lopera, Juan G. 565

López, Ruth 671

López-Moreno, Hector Samuel 888

López-Vélez, Rogelio 489

Lorenzo, Marcelo Gustavo 109

Loroño, Rubén E. 1132

Loroño-Pino, Maria A. 1132

Lortholary, Olivier 303

Lozano-Fuentes, Saul 902

Lu, Po-Liang 141

Lu, Qunying 185

Lu, Xi 947

Luang-Suarkia, Dagwin 1145

Lucas, Carmen M. 511

Lum, Lucy Chai See 796, 936

Luquetti, Alejandro O. 267

Lv, Yanli 306

Lye, David C. 1116

\section{M}

MacLeod, William B. S105

Maco, Vicente 781

Madeira, Maria de Fátima 257

Magak, Ng'wena G. 806

Magalhães, Belisa M. L. 1119

Magan, Christina 399

Magill, Alan 192

Magne, Denis 1041

Magnusson, Ulf 1076

Magumba, Godfrey S46

Mahgoub, Hyder 35

Maiga, Amelia W. 23

Makumbi, Frederick S97

Makundi, Rhodes H. 319

Malambo, Thindo 57

Maleewong, Wanchai 706

Marcos, Luis A. 491

Marín, Clotilde 481

Maro, Venance P. 1105

Maron, Angela 371

Marquart, Louise 63

Marques, Alexandre F. 87

Marques-da-Silva, Silvia Helena 710

Marsh, David R. S2, S6, S46, S69, S77, S151

Martín-Nalda, Andrea 832

Masaninga, Freddie 57

Massougbodji, Achille 418

Matey, Elizabeth 874

Matey, Elizabeth J. 1065

Mathieu, Els 216

Mathison, Blaine 297

Mathison, Blaine A. 1073

Mayxay, Mayfong 171

Mbonye, Anthony K. S46

McAuliffe, Isabel 297

McBride, Colleen M. 623

McCarl, Victoria 743

McCarra, Matthew 806

McCarty, Kathleen M. 979

McGorman, Laura S69

McGready, Rose 35

McGready, Rose 447

McManus, Donald P. 972

McQuiston, Jennifer H. 529
Meek, Sylvia R. S111

Mehrotra, Sanjana 655

Mehta, Rajesh D. 261

Melo, Gisely C. 1119

Meltzer, Eyal 264

Mendoza-Hernández, Guillermo 888

Menezes, Maria José 813

Mens, Petra F. 251

Meshnick, Steve 783

Meshnick, Steven R. 29, 1022

Messouri, Ibtissam 481

Mgalula, Leslie S54

Michaeli, Shulamit 264

Michels, Meta 943

Miller, E. Nancy 663

Miller, John M. 437

Minda-Aluisa, Elizabeth 524

Miri, Emmanuel 272

Mishra, Akhilesh C. 576

Misse, Dorothée 504

Mitchell, Shira 850

Mo, Ziyao 947

Modabber, Farrokh 70

Modibo, Bamadio S77

Moe, Christine 385

Moen, Ann 965

Mohamed, Mohd Sapian 737

Mohamud, Amina Issa S144

Mohebali, Mehdi 70

Mojtahedzadeh, Mona 162

Molina, Diego A. 1094

Molina, Douglas M. 460

Molina, Israel 832

Molina, Ricardo 1028

Molyneux, Malcolm E. 985

Monache, Luca Delle 902

Monaghan, Andrew J. 902

Montano, Silvia M. 954

Monteon, Victor 671

Montgomery, Susan P. 297, 862, 874, 1038, 1065

Montilla, Marleny 837

Montoya, Romeo H. 584

Moonga, Hawela 437

Moore, Catrin E. 171

Morais, Lorena 822

Moreira, João Soares 257

Moreno-Román, Paola 153

Morrissey, Anne B. 1105

Mota, Theo 109

Mouchet, François 504

Moura e Castro, Felipe A. 87

Mourão, Maria P. G. 1119

Mourya, Devendra T. 576

Moustafa, Manal 312

Mtimuni, Angella S54, S61

Muchiri, Eric M. 1059

Muchiri, Geoffrey 874

Mukanga, David S21

Mukhtar, Maowia M. 1012

Mukund, Amar 1

Mulligan, Mark J. 1112

Mulu, Moges 128

Mulu-Droppers, Ruth 128

Mundal, Kirk D. 511

Mungai, Peter L. 1059

Munkhjargal, Tserendorj 681

Muñoz, Maribel 399

Muok, Erick M. 862

Murillo, Carolina 1094

Murphy, Darby 565

Murta, Silvane M. 87

Mutebi, John-Paul 1125

Mwapasa, Victor 29, 1022 
Mwinzi, Pauline M. N. 862, 874, 1065 Mworozi, Edison S30

\section{$\mathbf{N}$}

N'Goran, Eliézer K. 425

N'Guessan, Raphael 242

Nada, Rania A. 312

Nakhla, Isabelle 312

Nakirunda, Maureen S97

Nanda, Kavita 743

Nanyonjo, Agnes S97

Nasr, Ahmed 701

Navarro, Miriam 489

Naveca, Felipe G. 1119

Nawa, Yukifumi 706

Nayyar, Gaurvika M. L. 231

Naze, Florence 134

Ndiaye, Daouda 640

Ndiaye, Jean Louis 640

Ndiaye, Magatte 640

Nefdt, Rory S85

Nelson, Adrianne K. 399

Nemeth, Nicole 760

Nevin, Remington L. 957

Newton, Paul N. 171, 573

Ng, Caroline L. 16

Ng, Chiu Wan 796

Ngoc, Tran Van 165

Nguyen, Hong Van 989

Nguyen, Yen T. 631

Nguyet, Nguyen Minh 165

Niamké, Sébastien 425

Niborski, Leticia L. 267

Nichol, Stuart T. 576

Nicholls, Rubén S. 837

Nielsen, Henrik V. 1046

Niezgoda, Michael 206

Njenga, Sammy M. 1004

Nkhoma, Ella T. 29, 1022

Noel, Megan S120

Nogara, Paula R. B. 1149

Nonvignon, Justice S11

Noor, Abdisalan M. 1004, 1012

Norbis, Walter 1083

Norman, Francesca F. 489

Nosten, François 35, 447

Nowosiwsky, Andry 960

Nsona, Humphreys S54, S61, S127

Null, Clair 786

Nurleila, Siti 41

Nyamjargal, Tserendorj 681

Nygren, Benjamin 594

\section{$\mathbf{O}$}

O'Connor, Annette 822

O'Reilly, Laura 407

O'Rourke, Peter 631

Obure, Alfredo 594

Ocampo, Martha C. 565

Ocan, Charles S46

Ochoa-Martinez, Carolina 902

Odhiambo, Keziah 862

Oduro, Abraham R. S21

Oesterle, Paul 760

Oga, Enoche F. 64

Ogutu, Michael 1065

Olivera, Paula 153

Olmo, Francisco 481

Omedo, Martin O. 1065

Ooi, Eng Eong 1116

Orellana, E. Roberto 954

Orjuela-Sánchez, Pamela 813
Orlandi, Renata 675

Ortiz, Sylvia 473

Oshiro, Elisa T. 470

Osikowicz, Lynn M. 518

Osorio, Jorge E. 565

Otero, Susana 832

Otieno, Ronald 594

Otoukesh, Salman 162

Ouédraogo, Smaïla 418

Oztel, Olga Nehir 81

\section{P}

Pagano, Marcello 850

Pagnoni, Franco S2, S11, S21, S120, S151

Pahl, Michelle K.

Palaniswamy, Shanmuga Sundaram 963

Panella, Amanda J. 1112

Pape, Jean W. 202

Pariyo, George S30

Partap, Uttara 11

Patel, Minal K. 594

Patil, Anand P. 1012

Patil, Sumeet 18

Patra, Kailash P. 412

Pattanayak, Subhrendu K. 18

Paulo Arruda, Paulo 822

Pavía, Paula 837

Peck, Robert N. 868

Peng, Ying 306

Pérez, Ana B. 538

Pérez-Molina, José Antonio 489

Perkins, Mark D. 202

Perry, Henry B. S144

Pesaresi, Alessandro 153

Petersen, Brett W. 206

Petersen, Christine A. 822

Peterson, Stefan S2, S36, S69, S92, S151

Phommasack, Bounlay 965

Phonekeo, Darouny 965

Picardeau, Mathieu 134

Piedrahita, Leidy D. 565

Pilingana, Portipher S105

Pimanpanarak, Mupawjay 447

Pimentel, Guillermo 312

Pimentel, Maria Inês Fernandes 257

Pinzón, Miguel Alejandro 195

Piper, Amanda 743

Plowe, Christopher V. 23, 231

Pomeroy, Amanda S120

Pongchaiyakul, Chatlert 706

Ponnampalavanar, Sasheela A. 936

Pontes, Nubia N. 663, 822

Poorabdollah, Mihan 162

Prajapati, Vijay Kumar 655

Prasad, Neha 261

Proux, Stephane 447

Puerta, Concepción J. 837

\section{$\mathbf{Q}$}

Qadri, Firdausi 921

Qazi, Shamim S137

Qi, Shun-Xiang 364

Quashie, Neils B. 996

Quick, Robert 594

Quiles, Michelle 743

Quyen, Nguyen Than Ha 165

Qvarnstrom, Yvonne 715, 1038

\section{$\mathbf{R}$}

Raboni, Sonia M. 371

Raeisi, Ahmad 499
Rahman, Jamalludin Ab 737

Rahman, Md. Ajijur 658

Rahvari, Seyed K. 162

Rai, Madhukar 655

Rai, Shailesh 18

Ramalingam, Rajalingam 737

Ramirez, Mercy G. 292

Ramírez-Macías, Inmaculada 481

Ramos-Ligonio, Angel 671

Raso, Giovanna 972

Raut, Chandrashekhar G. 576

Recuenco, Sergio 206, 491, 518

Reis, Mitermayer G. 843

Reis, Renato Barbosa 843

Reisen, William K. 559

Remoue, Franck 504

Restrepo, Angela 1094

Restrepo, Carlos A. 1094

Rezaee, Mohammad A. 70

Rheingans, Richard 385

Riazirad, Farhad 70

Ribeiro, Geyza Machado 741

Ribeiro, Mariana 743

Ribolla, Paulo E. M. 470

Richards Jr., Frank 272

Richards-Kortum, Rebecca R. 223

Righetti, Aurélie A. 425

Rijken, Marcus J. 447

Ringwald, Pascal 231

Ríos, Lyda C. 837

Rios, Maria 548

Ríos-Sicairos, Julian 888

Ritchie, Scott 1153

Rivera, Patricia 345

Rivière, Sébastien 303

Rivitti, Evandro A. 675

Roca, Yelin 504

Rocha, Marcele N. 87

Roche, Ingrid 1083

Rodrigues, Anderson Messias 710

Rodriguez, Lourdes 897

Rodriguez, Mary L. 292

Rodriguez, Silvia 897

Rogerson, Stephen J. 29, 1022

Rogier, Christophe 504

Rolfes, Melissa A. 806

Rolling, Thierry 190

Romanha, Alvaro J. 87

Ron-Román, Jorge 524

Rosell, Gustavo 399

Rosero, Doris A. 910

Rowland, Mark 242

Rubio, Giselia 371

Rueda, Daniel 153

Ruiz, Stefani Sesler 242

Rupprecht, Charles 206, 518

Rutebemberwa, Elizeus S30, S36

Rwiyereka, Angelique K. 581

Ryan, Edward T. 921

Ryan, Peter A. 554, 631

Saboori, Shadi 385

Sadruddin, Salim S77, S137

Sadumah, Ibrahim 594

Saegerman, Claude 524

Saganda, Wilbrod 1105

Sagara, Issaka 23, 50

Saha, Nirod Chandra 921

Saiyasombat, Rungrat 1132

Sajadi, Mohammad M. 162

Salant, Harold 883

Saldivar, Judith 399 
Salgueiro, Mariza de Matos 257

Sambo, Yohanna 272

Samuels, Aaron M. 874

San Martín, José L. 584

Sánchez-Moreno, Manuel 481

Sanjaya, Guardian Yoki 1152

Sanogo, Kassim 23

Sanogo, Kassim 50

Sanpool, Oranuch 706

Santos, Sara L. 87

Satoskar, Abhay R. 261

Saunderson, Paul R. 327

Schallig, Henk D. F. H. 251

Schijman, Alejandro G. 267

Schmolke, Kathrin 538

Schubach, Armando de Oliveira 257

Schuler, Douglas A. 602

Schurer, Janna M. 495

Schwalfenberg, Stefan 125

Schwartz, Eli 264

Sebastian, Jose Luis 399

Sebastian, Silvie 943

Sebeny, Peter J. 312, 996

Secor, W. Evan 862, 874, 1065

Segovia, Verónica 473

Seidenberg, Philip D. S105

Sekaran, Shamala Devi 936

Sekeseke-Chinyama, Masela 57

Sekine, Shuichi 64

Sengendo, Hanifah Naamala S46

Shabani, Aliakbar 70

Shahandeh, Khandan 499

Shahsafi, Mohammad R. 162

Shan, Xiaoxiao 947

Sheen, Patricia 153

Shehzad, Shafqat S137

Shepard, Donald S. 581, 796

Shete, Anita M. 576

Shi, Lei 947

Shi, Yonglin 185

Shin, Sonya 399

Shitara, Yoshihisa 64

Shu, Pei-Yun 349

Siazeele, Kazungu S105

Siba, Peter Max 1145

Sichamba, Bias S77

Sidi, Yechezkel 264

Sidibe, Bakary 23, 50

Sierra, Beatriz 538

Silpapojakul, Khachornsakdi 76, 927

Silpasakorn, Saowaluk 148

Silva, Fátima da Conceição 257

Silveira-Gomes, Fabíola 710

Silverman, Rachel 579

Sima, Laura C. 979

Simmons, Cameron P. 165

Simon, Zaira Barja 504

Sims, Annette C. 1073

Siqueira, André M. 1119

Sirima, Sodiomon B. S21

Siriyasatien, Padet 76

Sivakumar, Thillaiampalam 681

Smith, Katherine M. 743

Smith, Kirk 1125

Snow, Robert W. 1004, 1012

Soares, Rodrigo P. 87

Sobsey, Mark D. 394

Solari, Aldo 473

Solis, José 153

Sotelo, Jun 153

Sousa Jr., Joaquim X. 675

Sousa-Figueiredo, José Carlos 694

Souza, Raquel P. 1149

Souza, Samaly S. 843
Spears, Carla J. 743

Sripa, Banchob 281

Srisamut, Nujorn 148

Ssali, Sarah S36

Ssekitooleko, James S46

Staples, J. Erin 179, 1112

Steinhardt, Laura 192

Steinhoff, Daniel F. 902

Steinlauf, Shmuel 264

Stella, Vanessa 371

Stenos, John 342

Stensvold, C. Rune 1046

Stober, Carmel B. 663

Stoddard, Robyn A. 1105

Stone, Christopher M. 727

Stothard, J. Russell 694

Strachan, Clare S46

Strachan, Daniel L. S111

Stramer, Susan L. 548

Strickman, Daniel 719

Su, Chien-Ling 349

Subramanyam, Padma 963

Sulleiro, Elena 832

Sun, Benjamin 297

Sundar, Shyam 655

Sundaram, Lakshmi 202

Suputtamongkol, Yupin 148

Susan Lees, Rosemary 796

Suy, Anna 832

Swedberg, Eric S77

Syafruddin, Din 41

Sylla, Mariame S85

\section{T}

Tachibana, Hiroshi 97

Tadele, Getnet 623

Tahbaz, Mohammad O. 162

Tahita, Christian M. 251

Takagi, Hidekazu 658

Takesue, Atsuhide 658

Takken, Willem 242

Talbot, Elizabeth A. 202

Tam, Pham Thi 554

Tan, Esterlina V. 327

Tanwar, Ram K. 261

Taojaikong, Thaksinaporn 171, 573

Tapia-Santos, Berenice 902

Tarning, Joel 35

Tasso Jr., Norton 470

Tavares, Michel A. 1119

Taylor, Lizeth 345

Taylor, Terrie E. 985

Teixeira, Bernardo R. 371

Teixeira, Jorge J. V. 1149

ten Asbroek, Augustinus H. A. S111

Terkawi, Mohamad Alaa 681

Tesh, Robert B. 1132

Thanchomnang, Tongjit 706

Thang, Ngo Duc 989

Thellier, Marc 1041

Thera, Mahamadou A. 23

Thevanesam, Vasanthi 342

Thi, Xuan Cuc Pham 554

Thomas, Gwynneth S. 473

Thomas, Malcolm E. 743

Thompson, R. C. Andrew 281

Thongpaseuth, Soulignasack 171, 573

Thu, Ho Thi Viet 1076

Tibenderana, James S111

Tibenderana, James K. S21

Tine, Roger 640

Tinto, Halidou 251

Tiono, Alfred B. S21
Tobón, Angela M. 1094

Tognim, Maria C. B. 1149

Togo, Amadou 50

Tomson, Göran S97

Tora, Abebayehu 623

Torrecilhas, Ana C. 87

Torres-Pérez, Fernando 473

Touafek, Feriel 1041

Toure, Sekou 23, 50

Towner, Jonathan S. 576

Townsend, John 1125

Tram, Ta Van 165

Tran, Hau P. 631

Traore, Oumar Bila 23

Traoré/Coulibaly, Maminata 251

Tsai, Jih-Jin 141

Tsuyuoka, Reiko 965

Tu, Tran Cong 554

Tung, Nguyen Thanh 165

Turbes, Anna 786

\section{$\mathbf{U}$}

Uchimura, Nelson S. 1149

Uddin, Taher 921

Uejio, Christopher K. 902

Umar, Imram 715

Umaru, John 272

Umezawa, Eufrosina S. 675

Undurraga, Eduardo A. 796

Unnasch, Thomas R. 1140

Utzinger, Jürg 425

\section{V}

Valdivia, Hugo O. 511

Valete-Rosalino, Cláudia Maria 257

Vallois, Dorothée 1041

van Dam, Govert J. 868

van den Eede, Peter 989

van der Ven, André J. A. M. 943

van der Zee, Janneke 583

van Lieshout, Lisette 868

van Overmeir, Chantal 989

Vargas-Rodríguez, Rosa del Carmen Miluska 813

Vasconcellos, Érica de Camargo Ferreira e 257

Vélez, Iván Dario 1028

Vennerstrom, Jonathan L. 231

Vera, Hubert 511

Versteeg, Inge A. J. 251

Vieira, Vivaldo Pim 1089

Villahermosa, Laarni G. 327

Villamizar, Katherine 837

Vinetz, Joseph M. 196, 412

Visser, Benjamin Jelle 583

Visvesvara, Govinda S. 715

Vogt, Katrin 538

Volk, Hans-Dieter 538

Vongphrachanh, Phengta 965

Vongxay, Khamphouth 281

Vourc'h, Gwenaël 134

\section{W}

Waiswa, Peter S46

Walsh, Douglas S. 327

Walsh, Gerald P. 327

Walter, Annie 504

Wamani, Henry S92

Wang, Boliang 947 
Wang, Guangze 646

Wang, Peiyu 306

Wang, Ruobing 460

Wang, Seok Mui 936

Wang, Shanqing 646

Wang, Shuo 1053

Wang, Xian-Jun 1099

Wansi, Emmanuel S69

Watanabe, Emi 623

Wateska, Angela R. 616

Webster, Duncan 715

Weeks, John R. 608

Wegmüller, Rita 425

Wei, Ya-Mei 364

Weil, Ana A. 921

Weil, Gary J. 491

Weinstein, Philip 1153

Weitzel, Thomas 190

Wellems, Thomas E. 231

Welsh-Rodriguez, Carlos 902

Wen, Hao 104

Were, Vincent 594

Wettstein, Zachary S. 616

Wheeler, Sarah S. 559

White, A. Clinton 128

White, Gregory S. 1140

White, Nicholas J. 785

White, Valerie A. 327

Wiegand, Ryan E. 874

Wijesundara, Sarojini 342

Wilcox, Bruce A. 337

Wilkins, Patricia P. 297

Williams, Timothy S120

Willms, Kaethe 888
Willoughby Jr., Rodney E. 205

Wills, Bridget 165

Witt, Lars 125

Wittcoff, Alison S144

Wolfheim, Cathy S6

Wong, Mayee 337

Woods, Leslie 559

Worrell, Caitlin 216

Wu, Chi-Jung 141

Wu, Kejian 646

Wu, Liang 97

$\mathbf{X}$

Xiao, Dan 646

Xiao, Ning 1053

Xu, Bianli 185

$\mathrm{Xu}$, Dezhong 646

$\mathrm{Xu}$, Jianguo 185

$\mathrm{Xu}$, Longqi 97

$\mathrm{Xu}$, Yong-Gang 364

\section{Y}

Yadav, Pragya D. 576

Yamanija, Jose 399

Yan, Yongping 646

Yang, Bin 97

Yang, Cheng-Fen 349

Yang, Hai 689

Yang, Titi 306

Yang, Xiangliang 689
Yangzom, Thinley 1028

Yeboah-Antwi, Kojo

Yeboah-Antwi, Kojo S77, S105

Yesilkir-Baydar, Serap 81

Yokoyama, Naoaki 681

Young, Ginger 1125

Young, Mark S6, S85

Young, Sylvia Y. N. 312

Yu, Qin 689

Yu, Xue Jie 185

Yukich, Joshua O. 437

\section{$\mathbf{Z}$}

Zamasiya, Texas S54

Zambrano, Betzana 584

Zanko, Rodrigo L. 1149

Zeng, Wu 581

Zhang, Lijuan 185

Zhang, Meng 1099

Zhang, Xiuchun 185

Zhang, Yan-Bo 364

Zhang, Yumei 306

Zhang, Zhiwen 148

Zhao, Ai 306

Zhao, Hongbo 947

Zhao, Zhong-Tang 1099

Zheng, Hong 104

Zhu, Yanhong 689

Zielinski-Gutierrez, Emily 902

Zimic, Mirko 153

Zorrilla, Victor O. 511

Zunt, Joseph R. 954 